OPEN ACCESS

Edited by:

Katrin Schäfer,

Johannes Gutenberg University Mainz,

Germany

Reviewed by:

Elena Kaschina,

Charité - Universitätsmedizin Berlin,

Germany

Luca Valerio,

Johannes Gutenberg University Mainz,

Germany

*Correspondence:

Jonathan Golledge

Jonathan.Golledge@jcu.edu.au

Specialty section:

This article was submitted to Translational Endocrinology,

a section of the journal

Frontiers in Endocrinology

Received: 20 October 2020 Accepted: 12 January 2021 Published: 15 March 2021

Citation:

Thanigaimani S and Golledge J (2021) Role of Adipokines and Perivascular Adipose Tissue in Abdominal Aortic Aneurysm: A Systematic Review and Meta-Analysis of Animal and Human

Observational Studies.

Front. Endocrinol. 12:618434. doi: 10.3389/fendo.2021.618434

\section{Role of Adipokines and Perivascular Adipose Tissue in Abdominal Aortic Aneurysm: A Systematic Review and Meta-Analysis of Animal and Human Observational Studies}

\author{
Shivshankar Thanigaimani ${ }^{1,2}$ and Jonathan Golledge $\mathrm{e}^{1,2,3^{*}}$ \\ 1 The Queensland Research Centre for Peripheral Vascular Disease (QRC-PVD), College of Medicine and Dentistry, James \\ Cook University, Townsville, QLD, Australia, ${ }^{2}$ The Australian Institute of Tropical Health and Medicine, James Cook \\ University, Townsville, QLD, Australia, ${ }^{3}$ The Department of Vascular and Endovascular Surgery, Townsville University \\ Hospital, Townsville, QLD, Australia
}

Improved understanding of abdominal aortic aneurysms (AAA) pathogenesis is required to identify treatment targets. This systematic review summarized evidence from animal studies and clinical research examining the role of adipokines and perivascular adipose tissue (PVAT) in AAA pathogenesis. Meta-analyses suggested that leptin (Standardized mean difference [SMD]: 0.50 [95\% confidence interval (Cl): -1.62, 2.61]) and adiponectin (SMD: -3.16 [95\% Cl: $-7.59,1.28]$ ) upregulation did not significantly affect AAA severity within animal models. There were inconsistent findings and limited studies investigating the effect of resistin-like molecule-beta (RELM $\beta$ ) and PVAT in animal models of AAA. Clinical studies suggested that circulating leptin (SMD: 0.32 [95\% Cl: 0.19, 0.45]) and resistin (SMD: 0.63 [95\% Cl 0.50, 0.76]) concentrations and PVAT to abdominal adipose tissue ratio (SMD: 0.56 [95\% Cl 0.33, 0.79]) were significantly greater in people diagnosed with AAA compared to controls. Serum adiponectin levels were not associated with AAA diagnosis (SMD: -0.62 [95\% Cl -1.76, 0.52]). One, eight, and one animal studies and two, two, and four human studies had low, moderate, and high risk-of-bias respectively. These findings suggest that AAA is associated with higher circulating concentrations of leptin and resistin and greater amounts of PVAT than controls but whether this plays a role in aneurysm pathogenesis is unclear.

Keywords: adipokine, adipose tissue, periaortic adipose tissue, abdominal aortic aneurysm, aortic rupture

\section{INTRODUCTION}

Screening studies suggest that abdominal aortic aneurysms (AAA) prevalence is approximately $3 \%$ in men and $1 \%$ in women aged over 65 years (1). The main complication of AAA is aortic rupture which is estimated to cause 200,000 deaths worldwide each year (2). Currently the management of AAA is limited to surgical repair (3). Randomized clinical trials have demonstrated that early elective surgical repair does not reduce mortality for people with small asymptomatic AAAs $(4,5)$. 
Current guidelines therefore recommend simply monitoring small $(<50 \mathrm{~mm}$ in women and $<55 \mathrm{~m}$ in men) asymptomatic AAAs (6). Most small AAAs continue to expand and eventually reach a diameter when surgical treatment is indicated (6). Drug therapies which limit growth of small AAAs would be valuable in clinical practice. Previous small randomized controlled trials have however failed to identify any effective medications (7).

Adipocytes are fat depots that store excess energy as triglycerides. They also have an endocrine function by secreting adipokines, such as leptin, adiponectin, dipeptidyl peptidase-4 (DPP-4), resistin and resistin-like molecule beta (RELM $\beta$ ) (8). Leptin is an adipokine involved in regulating energy homeostasis and obesity. It is considered an important regulator of $\beta$ cell mass and survival (9). In patients with leptin deficiency, recombinant leptin replacement therapy suppresses appetite and increases energy expenditure and leptin has been developed as a treatment for obesity (10). The adipokine adiponectin has insulin-sensitizing, anti-inflammatory and anti-apoptotic effects (11). Adiponectin and agonists of its receptor have also been suggested as a treatment for obesity and its complications (12). DPP4 release has been shown to strongly correlate with adipocyte size, potentially representing an important source of DPP4 in obesity (13). Resistin belongs to an RELM family and is believed to be an important link between obesity, insulin resistance, and diabetes (14). Increasing evidence suggests that resistin plays an important role in a variety of biological processes involved in cardiovascular diseases, autoimmune disease, and asthma (14). RELM- $\beta$ is another member of RELM family that is mainly secreted from the adipose tissue in rodents (15) and from adipose-associated macrophages in humans (16). Adipose is deposited over multiple subcutaneous and visceral sites but when considering a role in aortic pathology, perivascular aortic adipose tissue (PVAT) is of particular interest. Adipose tissue residing in the vascular adventitia has been proposed to act like endocrine cells that respond to inflammatory stimuli by releasing adipokines and other signaling mediators to maintain vascular homeostasis and potentially play a role in aortic pathology $(17,18)$. This review summarizes published animal and human studies that investigated the role of these adipokines and PVAT in AAA pathogenesis.

\section{METHODS}

\section{Identification and Inclusion of Studies in This Review}

Studies were identified from the PubMed database published until $5^{\text {th }}$ August 2020 using the following search terms ["adiposity" OR "adipose tissue" OR "adipokines" OR "adiponectin" OR "leptin" OR "resistin" OR "subcutaneous fat" OR "visceral fat" OR "peri aortic fat" OR "obesity"] AND ["Abdominal aortic aneurysm" OR "aortic occlusive disease" OR "aortic rupture"]. Animal and observational human studies that investigated the role of adipokines or PVAT in AAA were included. Outcomes included aortic diameter and adipokine concentrations or relative amount. Included articles were identified by one author (ST) and reviewed by another author (JG). A minimum of two studies reporting the aortic diameter in animal studies and adipokines levels or PVAT in human studies were required for meta-analyses to be performed. In studies where the effect of deficiency and inhibition of a specific adipokine or PVAT were reported, data were converted to an equivalent format in order to include in the same meta-analyses. Meta-analysis findings were presented as standardized mean difference (SMD) and 95\% confidence intervals (CI). Metaanalyses were performed using random effects model and an inverse variance method was used to estimate the heterogeneity between studies. All analyses were conducted using the "meta" package of $\mathrm{R}$ software version 3.4.4. $\mathrm{p}$ value of $\leq 0.05$ were considered significant.

The quality of the animal studies was assessed using a modified version of the Animal Research: Reporting of In Vivo Experiments (ARRIVE) guideline criteria. The quality of the included human studies was assessed using the modified version of Newcastle-Ottawa Scale 10 which assessed the study design, sample size estimation, age and sex-matching of controls, whether imaging was performed to measure AAA size, method of AAA size assessment, adjustment for confounders, and whether the observers were blinded to group during analysis. Assessment scores with $<50,50-75$, and $>75 \%$ were considered to have high, moderate, and low risk of bias respectively.

\section{RESULTS AND DISCUSSION}

The initial search identified 383 studies. Based on the inclusion criteria, 321 studies were excluded due to not meeting the entry criteria. The full text of the remaining 62 studies (19 animal and 43 human) were reviewed of which ultimately 10 animal and 8 human studies were included (Figures 1 and 2).

\section{Animal Studies Investigating the Role of Adipokines or PVAT in AAA}

Ten eligible rodent studies were identified (19-28). These studies included a total of 319 rodents in which the effects of leptin, resistin, adiponectin, and PVAT were investigated (19-28). Detailed information about each study is shown in Table $\mathbf{1}$.

\section{Animal Studies Investigating the Effect of Leptin in $A A A$}

Four studies including 159 animals investigated the role of leptin in AAA pathogenesis within mouse model induced using Angiotensin-II (Ang-II) infusion $(19,21,22)$ or peri-aortic calcium chloride $\left(\mathrm{CaCl}_{2}\right)$ application (20) (Table 1A). These studies used mice with different backgrounds including Apolipoprotein E (ApoE) $(19,22)$, combined $\mathrm{Na}-\mathrm{Cl}$ cotransporter and interleukin (IL)-18 receptor (20), and low density lipoprotein receptor (LDL-r) (21) deficient mice. The role of leptin in AAA pathogenesis was explored through local peri-aortic application $(19,20)$, intraperitoneal injection (22), and gene deficiency $(20,21)$. 


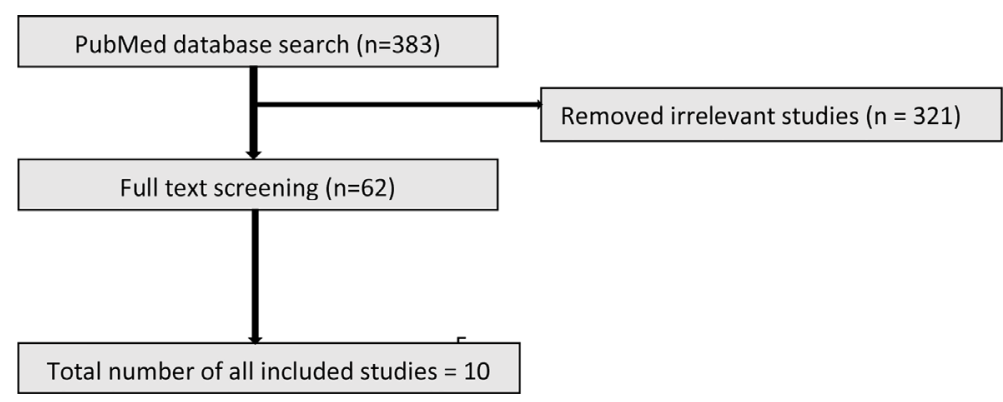

FIGURE 1 | PRISMA flow diagram illustrating the process of selection of the animal studies.

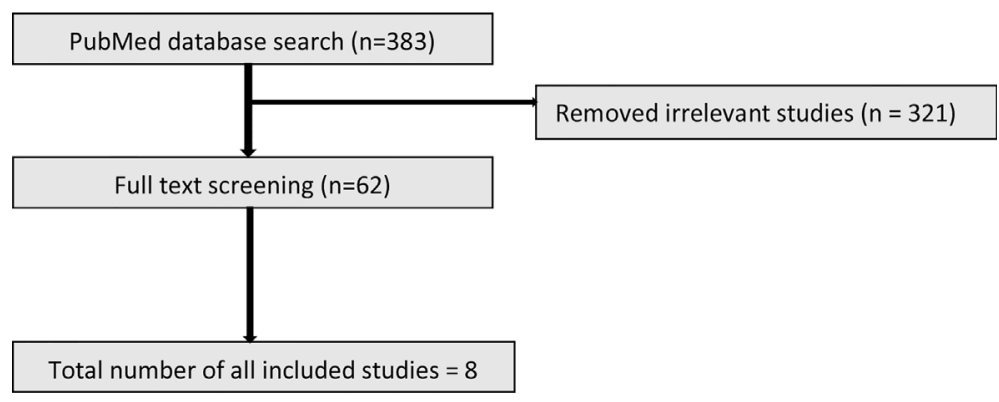

FIGURE 2 | PRISMA flow diagram illustrating the process of selection of the human studies.

Peri-aortic application of leptin was reported to promote a significant increase in AAA severity (diameter) in the Ang-II model (19). Leptin also promoted aortic and visceral adipose macrophage infiltration. A further study reported that perivascular implantation of white adipose tissue in obese or lean mice resulted in an increased AAA size over controls in the $\mathrm{CaCl}_{2}$ model (20). Perivascular implantation of adipose tissue from leptin deficient mice abolished the increase in AAA diameter size that was demonstrated when adipose tissue from leptin sufficient mice was used, suggesting a role of leptin in adipose tissue induced AAA development (20).

In contrast to the above findings, germline leptin deficiency has been reported to promote the risk of AAA rupture in obese mice infused with Ang-II but AAA size was not reported in this study (21). In keeping with a role of leptin in inhibiting AAA pathogenesis, intraperitoneal injection of leptin $(600 \mu \mathrm{g} / \mathrm{kg}$ daily) has been reported to reduce AAA diameter and risk of rupture in the Ang-II model (22). Leptin was reported to downregulate expression of the Th2 cytokine IL-4 and mRNA levels of GATA binding protein-3 (GATA-3), a key transcription factor for Th2 polarization. In addition, leptin also upregulated Th1 cytokine interferon-gamma (INF- $\gamma$ ) and T-box binding transcription factor (T-bet), a key transcription factor for Th1 polarization (22). These results led the authors to suggest that leptin could be a potential treatment for the prevention of AAA formation.

A meta-analysis of three eligible studies suggested that leptin upregulation had no significant effect on AAA diameter (SMD: 0.50 [95\% CI: $-1.62,2.61]$ ) (Figure 3). Overall the findings of these studies suggest that the effect of leptin depends on the method and site of up and downregulation. All four studies used the same mouse strain $(\mathrm{C} 57 \mathrm{BL} / 6)$ and experimental period but the method of leptin upregulation varied markedly which likely was the cause of the inconsistent findings. The studies reported that leptin promoted IL18 binding to inflammatory and vascular cells by upregulating the expression of IL18, the IL18-receptor, and the $\mathrm{Na}-\mathrm{Cl}$ co-transporter in addition to augmenting MMP-9 synthesis $(19,20,22)$.

\section{Animal Studies Investigating the Effect of Adiponectin on AAA}

Two studies including 64 animals investigated the effect of adiponectin on AAA development in mouse models $(23,24)$ (Table 1B). In high-fat fed mice with LDL-r deficiency, intravenous injection of a recombinant adenoviral vector encoding adiponectin was reported to inhibit AAA development in the Ang-II infusion model (23). Adiponectin was reported to inhibit AAA development by suppressing aortic inflammatory cell infiltration, medial degeneration, and elastin fragmentation (23). Adiponectin was also shown to inhibit the angiotensin type-1 receptor (AT1R), downregulate expression of inflammatory cytokines, mast cell protease, and adipose inflammation, and upregulate lysyl oxidase (LOX) expression in the aortic wall (23). In keeping with this, germline deletion of adiponectin has been reported to promote AAA development in the Ang-II infusion model (24). Adiponectin-deficiency augmented the early infiltration of macrophages and increased 
TABLE 1 | Animal studies investigating the role of adipokines and PVAT in AAA.

\begin{tabular}{lcccccc}
\hline Ref Animal strain $\quad$ Intervention & $\begin{array}{c}\text { AAA } \\
\text { model }\end{array}$ & Groups & $\begin{array}{c}\text { Sample } \\
\text { size }\end{array}$ & $\begin{array}{c}\text { Assessment } \\
\text { period }\end{array}$ & $\begin{array}{c}\text { Aortic } \\
\text { diameter } \\
\text { (mm) }\end{array}$ & Mechanisms implicated in AAA formation \\
\hline
\end{tabular}

A) Animal studies investigating the effect of leptin on aortic diameter

(19)

C57BL/6J mice Germline deficiency in Ang-II Control

(21)

leptin

High-fat

LDLr-/-

Leptin-/- Ob+

Leptin-/- Ob/Ob

(20)

57BL/6J mice Diet induced obesity $\mathrm{CaCl}_{2} \quad$ ApoE-/- no WAT control

ApoE-/- obese WAT

ApoE-/- lean WAT

ApoE-/- NcC-/- IL18r-/- 8

C57BL/6J mice I.P. injection

Ang-II Sham + Saline

(22)

Sham + Saline

Ang-II + leptin

28 days $\quad 1.5 \pm 0.1$ Para-visceral aortic leptin in $\mathrm{ApoE}^{-/-}$mice induces local medial degeneration and

$1.8 \pm 0.2$ augments angiotensin II-induced AAA.

28 days NR Leptin deficient obese mice exhibiting AAAs had greater macrophage content in

visceral adipose tissue than mice not developing AAA.

28 days $\quad 0.1 \pm 0.03$ Perivascular implantation of adipose tissue from either diet induced obese mice or

$1.1 \pm 0.5$ lean mice exacerbated AAA development, but this was abolished in leptin-deficient

$2.1 \pm 0.9$ obese mice.

$0.6 \pm 0.3$

28 days $\quad 1.0 \pm 0.2$ Pre-treatment with leptin significantly downregulated protein expression of the Th2

$2.3 \pm 0.6$ cytokine IL-4 and mRNA levels of GATA-3, the key transcription factor for Th2

$1.5 \pm 0.4$ polarization, and significantly upregulated Th1 cytokine INF- $\gamma$ and T-bet, the key transcription factor for Th1 polarization

B) Animal studies investigating the effect of adiponectin on aortic diameter

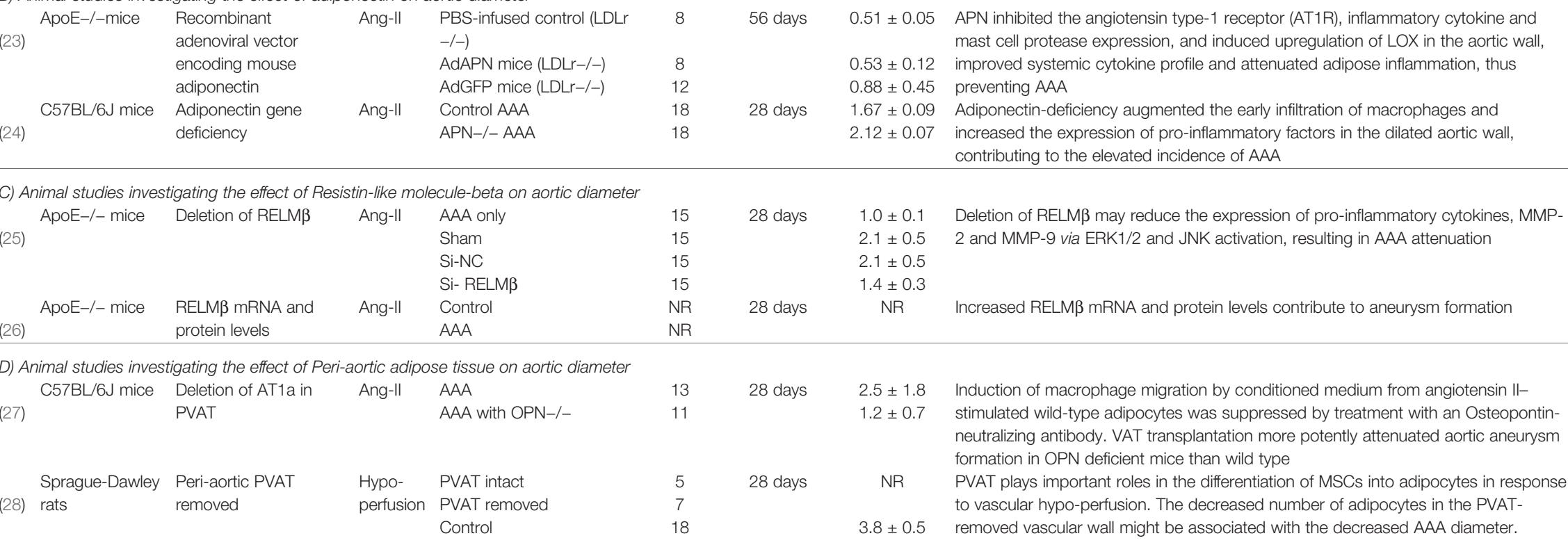

AAA, Abdominal aortic aneurysm; APN, Adiponectin; ADMSC, Adipose derived MSC; AdAPN, Recombinant adenoviral APN; AdGFP, Recombinant adenovirus green fluorescent protein; Ang, Angiotensin; ApoE, Apolipoprotein E; AT1a, Ang-ll type 1a; BMSC, Bone marrow derived stem cells; CaCl, Calcium chloride; CD, Clustered differentiation; G-CSF, Granulocyte colony stimulating factor; IL-18r, Interleukin 18 receptor; WT, Wild type; NCC, Na-Cl co-transporter; GATA, Erythroid transcription factor: HDL, High density lipoprotein; HF/HF. High-fat diet acclimatization for 20 weeks followed by high-fat diet continuation for another 8 weeks; HF/LF. High-fat diet acclimatization for 20 weeks followed by low fat diet for another 8 weeks; I.P., Intraperitoneal; INF, Interferon; IP-10, Interferon gamma-induced protein 10; LOX, Lysyl oxidase; LDL, Low density lipoprotein; LePA, Leptin antagonist; mRNA, Messenger Ribonucleic acid; MSC, Mesenchymal stem cells; MCP, Monocyte chemoattractant protein; MMP, Matrix metalloproteinase; NC, Negative control; NA, Not available; NR, Not reported; OPN, Osteopontin; ob/ob, Obese mice; PLGA, Poly lactic- co- glycolic acid; PVAT. Perivascular adipose tissue; RELMP Resistin-like molecule-beta; si, Small RNA inference; Th, Thelper cells; TG, Triglycerides; VAT, Visceral adipose tissue; WAT, White adipose tissue; ^Aortic lumen diameters not different, however, maximal diameters were significantly different. 


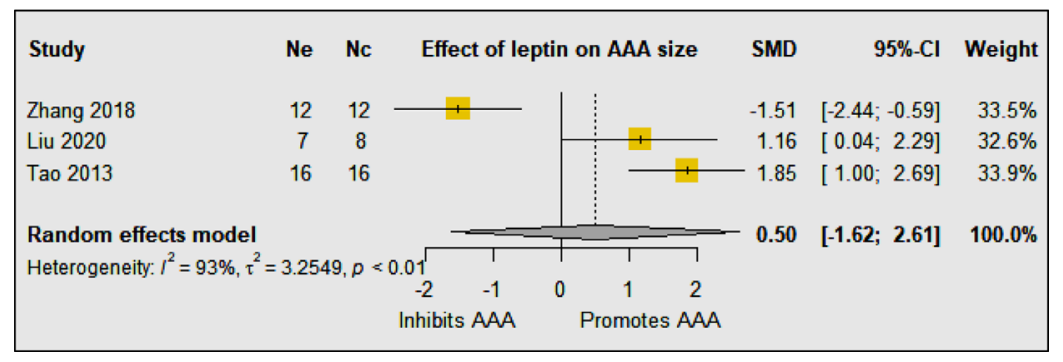

FIGURE 3 | The included studies had contrasting findings most likely due to the varied methods of leptin upregulation. The meta-analysis suggested that overall upregulation of leptin had no effect on AAA diameter. Ne, Number of animals in experimental group; Nc, Number of animals in control group.

the expression of matrix remodeling enzymes such as matrix metalloproteinase (MMP) -2 and 9 in the aortic wall. These results suggest that adiponectin protects against AAA formation through multiple different mechanisms, of which inhibiting inflammation plays a key role. Meta-analysis suggested that adiponectin upregulation did not significantly reduced AAA diameter (SMD: -3.16 [95\% CI: -7.59, 1.28]) (Figure 4). The findings of the meta-analysis need to be considered in the context of the limited number of available studies and included animals.

\section{Animal Studies Investigating the Effect of RELM $\beta$ on AAA}

Two studies including 60 mice in one study and an unreported number in one study investigated the role of RELM $\beta$ in AAA development $(25,26)$ (Table 1C). Both studies used the same mouse strain $\left(\mathrm{ApoE}^{-/-}\right)$and experimental period. Germline deletion of RELM $\beta$ resulted in significantly reduced AAA diameter within the Ang-II infusion model (25). This was associated with reduced macrophage accumulation and decreased expression of proinflammatory cytokines (monocyte chemoattractant protein 1 and IL6) and MMP2 and 9 in the aortic wall. Further, it has also been reported that RELM $\beta$ expression was upregulated within the aortas of mice with Ang-II induced AAA compared to controls (26). These limited investigations suggest that RELM $\beta$ could have a role in promoting AAA formation via induction of pro-inflammatory cytokines. Meta-analysis was not possible due to the lack of available studies.

\section{Animal Studies Investigating the Effect of PVAT on AAA}

Two studies reporting the effect of PVAT in AAA development in 54 animals were identified $(27,28)$ (Table 1D). In mice deficient in osteopontin, angiotensin II type 1a (AT1a) deficiency of PVAT significantly attenuated the size of AAA developing, macrophage infiltration, and gelatinolytic activity in the Ang-II model (27). Another study investigated the effect of AAA development following the removal of PVAT in a hypoperfusion induced AAA rat model (28). In this model, the authors intended to isolate the oxygen supply and induce hypoxia in the infra-renal aortic region. First, the PVAT was removed from around the infra-renal aorta to prevent oxygen supply via the adventitial vasa vasorum (VV). Following this, a polyurethane catheter was inserted via a small incision in the infra-renal aorta to separate the lumen blood flow from the aortic wall, thus isolating the oxygen supply to the aortic wall from the blood directly. These two steps reduced the oxygen supply and induced hypoxia in the infra-renal aortic region without affecting the systemic circulation, promoting AAA formation. It was reported that 28 days after the removal of the PVAT the number of CD44+ and CD90+ cells and adipocytes in the AAA wall was significantly less when PVAT had been removed. Mice that has PVAT removed were reported to have significantly smaller AAA diameter than controls (28). Evidence from other animal studies has suggested that PVAT contributes to intimal hyperplasia via leptin signaling (29). Samples from

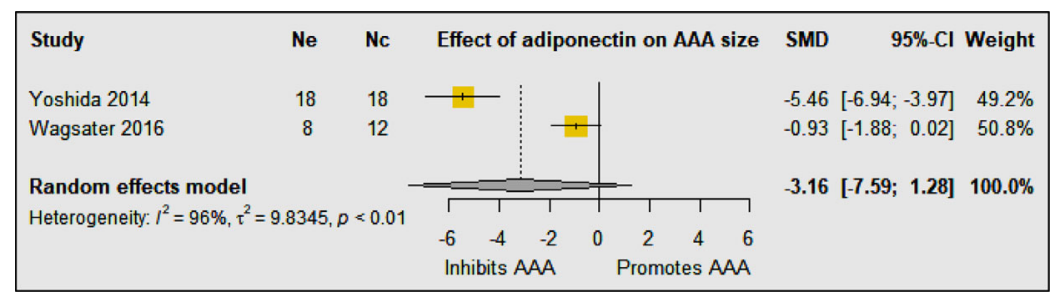

FIGURE 4 | Meta-analysis suggested that upregulation of adiponectin had no significant effect on AAA diameter. Ne, Number of animals in experimental group; Nc, Number of animals in control group. 
mouse models of AAA show infiltration by neutrophils, mast cells, and T-cells and expression of cathepsin $\mathrm{K}$ and $\mathrm{S}$ within PVAT which may contribute to AAA development (30). The exact role of PVAT in AAA development needs further investigation.

\section{Quality of Prior Animal Studies Investigating Adipokines and PVAT in AAA}

All studies reported an ethics approval statement, AAA model type and included relevant control groups (Table 2). None of the studies reported sample size estimates. Two studies reported that the assessors were blinded during the aortic diameter and/or adipokine assessments $(20,21)$. One study reported the reproducibility of aortic diameter assessment (20). Three studies did not report aortic diameter following the assessment period $(21,26,28)$. One study did not report either the age or weight of the animals used in the study (26). One study did not report the number of animals included in each group (26). Overall, one (20), eight $(19,21-25,27,28)$, and one (26) studies were considered to have low, moderate, and high risk of bias respectively.

\section{Evidence From Human Studies For A Role Adipokines and PVAT in AAA Pathogenesis Studies Investigating the Association of Adipokines With AAA Diagnosis}

Five studies were identified studying the association of circulating adipokines, including DPP-4, leptin, resistin, and adiponectin, with AAA in 1146 cases compared with 837 control participants (30-34) (Table 3). The design of the studies included retrospective cohort, case control, and prospective cohort. Two studies matched cases and controls for age $(33,34)$ and sex $(32,34)$. Three studies reported that controls were healthy $(30,32,34)$.

A case-control study reported that plasma DPP-4 enzyme activity was significantly increased in both large and small AAA patients compared to controls and positively correlated with AAA diameter (32) (Table 3A). However, circulating DPP-4 plasma levels were similar between control and small AAA participants and significantly lower in people with large AAA (32). This study suggested that increased DPP-4 activity may play a role in AAA pathogenesis but larger studies are needed.

Three studies including 1,034 AAA patients and 808 controls investigated the association of circulating leptin concentrations with AAA $(31,33,34)$ (Table 3B). A cohort sample of 701 patients from the Atherosclerotic Risk in Communities (ARIC) study suggested that plasma leptin levels were inversely associated with AAA diagnosis after adjusting for confounders including age, sex, race, smoking status, pack-years of smoking, height, hypertension, high and low density lipoproteins (HDL and LDL), total cholesterol, and peripheral artery disease, but the study did not report aortic diameter (31). A prospective cohort study showed that serum levels of leptin were similar in AAA and control participants after adjusting for age, smoking, body mass index (BMI), carotid artery stenosis, diabetes, hypertension, lipids, C-reactive protein (CRP), and statin prescription,

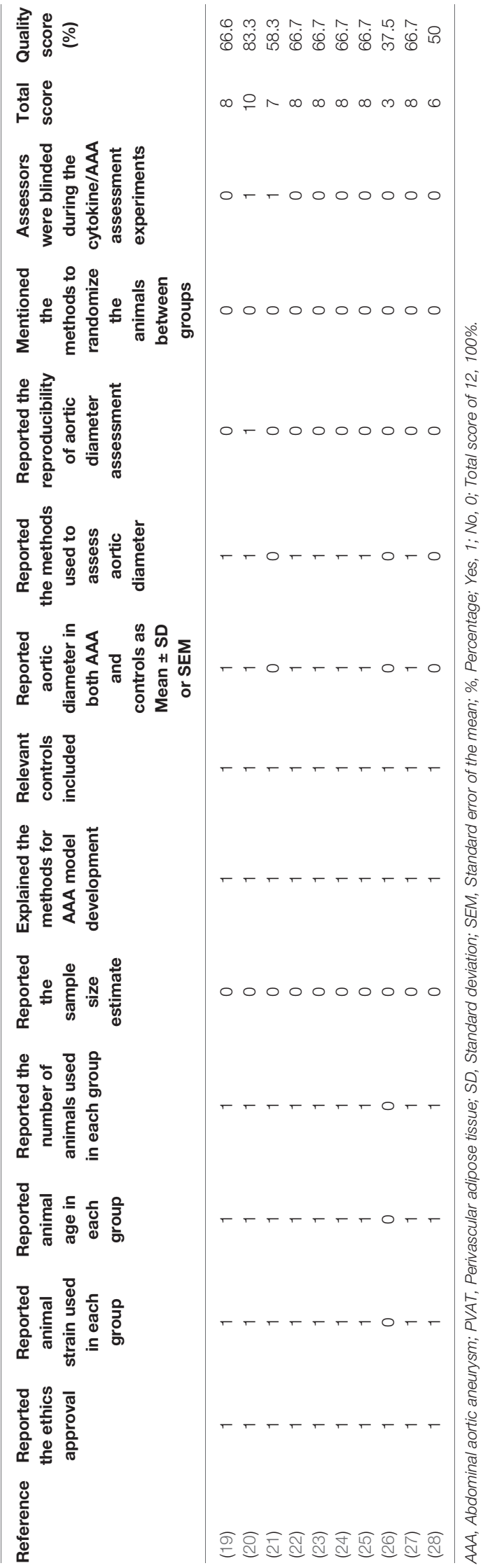


A) Studies estimating the association of DPP-4 activity and AAA

\begin{tabular}{ccccc} 
(32) $\quad$ Control & 20 & $\mathrm{NR}^{\$}$ & $9.9 \pm 9.2$ \\
& Small AAA & 16 & $3.6 \pm 0.7^{\$}$ & $19.2 \pm 8.2$ \\
& Large AAA & 77 & $5.9 \pm 1.4^{\$}(\mathrm{~cm})$ & $30.4 \pm 13.9$ \\
\hline
\end{tabular}

B) Studies estimating the association of leptin and AAA

(31) $\delta$ Retrospective cohort study AAA

(33) Prospective cohort study No AAA

AAA

(34) $\delta \quad$ Cohort from population based RCT Control AAA

70

174

$\begin{array}{r}634 \\ 318 \\ \hline\end{array}$

634
318

C) Studies estimating the association of Resistin and AAA

\begin{tabular}{lll} 
(33) $\quad$ Prospective cohort study & No AAA \\
& AAA \\
(34) $\delta$ & Cohort from population based RCT & Control \\
& & AAA \\
\hline
\end{tabular}

D) Studies estimating the association of adiponectin and AAA

(34) $\delta$

Prospective cohort study

AAA

$(30)^{\star \star}$

Cohort from population based RCT

Control

AAA

Control

AAA

174
15

634
318

174
15
634
318
9
19

$\begin{array}{cc}\text { NR } & \text { HR: } \\ & 0.8(0.7-1.0) \\ N R & 7.3(4.7-11.4) \\ N R & 9.7(4.9-17.3) \\ 9-22^{\#}(\mathrm{~mm}) & 12.6 \pm 9.9 \\ \geq 30(\mathrm{~mm}) & 16.5 \pm 16.7\end{array}$

$<0.05 \quad$ None

AAA, Abdominal aortic aneurysm; BMI, Body mass index; CHD, Coronary heart disease; Cl, Confidence interval; CRP, C reactive protein; cm, Centimeter; DM, Diabetes mellitus; DPP-4, Dipeptidyl peptidase-4; HR, Hazard ratio; HDL, High density lipoprotein; LDL, Low density lipoprotein; NR, Not reported; mm, Millimeter; PAD, Peripheral artery disease; RCT, Randomized controlled trial; TC, Total cholesterol; TG, Triglycerides; WHR, Waist to hip ratio; *Adipokine levels were either mentioned as mean \pm S.D. or median $\left(25^{\text {th }}-75^{\text {th }}\right.$ percentile range); *Adipokine levels data extracted from graph; 'Sub-analysis data assessed adipokines from a larger cohort; ${ }^{*}$ Aortic diameter measured using ultrasound (US); ${ }^{\$}$ Aortic diameter measured using Computed tomography (CT). 
suggesting that leptin was not associated with AAA (33). Another population based study reported leptin levels in 952 men screened for AAA, 318 of whom had an AAA diagnosed. Results from this study suggested that leptin levels were not independently associated with AAA after adjusting for confounders including age, smoking, BMI, carotid artery stenosis, diabetes, hypertension, HDL, LDL, total cholesterol, triglycerides, CRP, and statins (34). A meta-analysis suggested that overall circulating leptin levels were higher in people diagnosed with an AAA than controls (SMD: 0.32 [95\% CI: $0.19,0.45]$ ) (Figure 5). It was not possible to adjust this metaanalysis for confounding risk factors.

Two studies including 333 AAA patients and 808 controls investigated the association of serum resistin levels with AAA $(33,34)$ (Table 3 C). A prospective cohort study showed a trend toward higher resistin levels in participants with AAA compared to controls (33). Multivariate analyses showed that serum resistin levels were independently associated with AAA after adjusting for age, smoking, BMI, carotid artery stenosis, diabetes, hypertension, HDL, LDL, cholesterol, triglycerides, adiponectin, leptin, CRP, and statin medication (33). A population-based study in men found that serum resistin concentrations were independently associated with AAA after adjusting for age, dyslipidemia, hypertension, smoking, CHD, and diabetes mellitus, waist-hip ratio, and serum glucose (34). Meta-analysis of these studies suggested that serum resistin levels were significantly higher in people diagnosed with AAA compared to controls (SMD: 0.63 [95\% CI 0.50, 0.76]) (Figure 6).

Three studies including 352 AAA patients and 817 controls investigated the association of circulating adiponectin with AAA
$(30,33,34)$ (Table 3D). A prospective cohort study showed no independent association of serum levels of adiponectin with AAA after adjusting for age, smoking, BMI, carotid artery stenosis, diabetes, hypertension, lipids, CRP, and statin prescription (33). A population-based study found that serum levels of adiponectin were associated with AAA $\geq 30 \mathrm{~mm}$, but not AAA $\geq 40 \mathrm{~mm}$, suggesting that adiponectin may play a role in the early stages of AAA development (34). A case-control study reported that serum levels of adiponectin were significantly lower in AAA patients compared to healthy control organ donors, however, adiponectin was significantly increased in PVAT surrounding the AAA, suggesting that adipocytes surrounding the aorta may be a source of inflammatory leukocytes that are attracted by adipocytes undergoing necrosis and by proinflammatory ceramides (30). Meta-analysis suggested that serum adiponectin levels were not significantly associated with AAA diagnosis (SMD: -0.62 [95\% CI -1.76, 0.52]) (Figure 7). Phenotypic changes in PVAT resulting from vascular injury have been reported to promote neointimal formation via release of adipocytokines which in turn regulate inflammation, vascular smooth muscle cell proliferation, endothelial dysfunction, fibroblast activation and migration and neovascularization (35). Furthermore, high fat diet or smoking have also been suggested to promote vascular inflammation, reactive oxygen species production, and matrix degradation that augments AAA formation (36).

\section{Quality of Human Studies Investigating the Association of Adipokines With AAA}

All studies measured adipokines in AAA cases and a comparator group (Table 3) (30-34). Three studies reported the results after

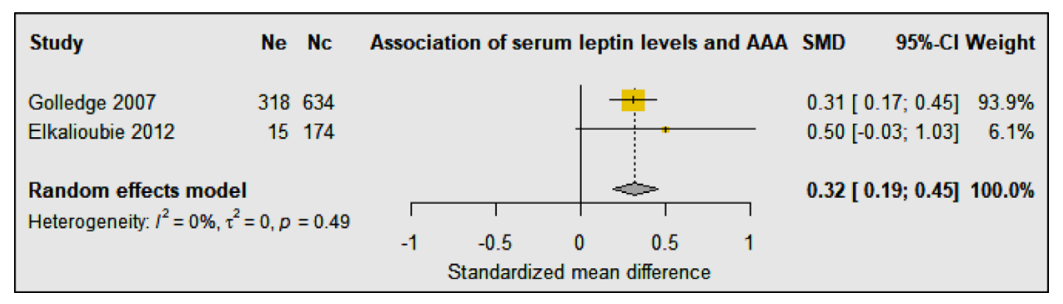

FIGURE 5 | Forest plot suggesting the association of high circulating leptin concentrations with AAA diagnosis. Ne, Number of patients in experimental group; Nc, Number of patients in control group.

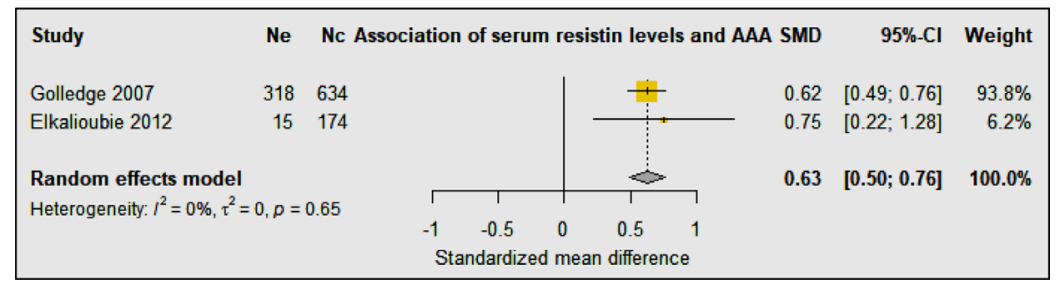

FIGURE 6 | Forest plot suggesting the association of high circulating resistin concentrations with AAA diagnosis. Ne, Number of patients in experimental group; Nc, Number of patients in control group. 


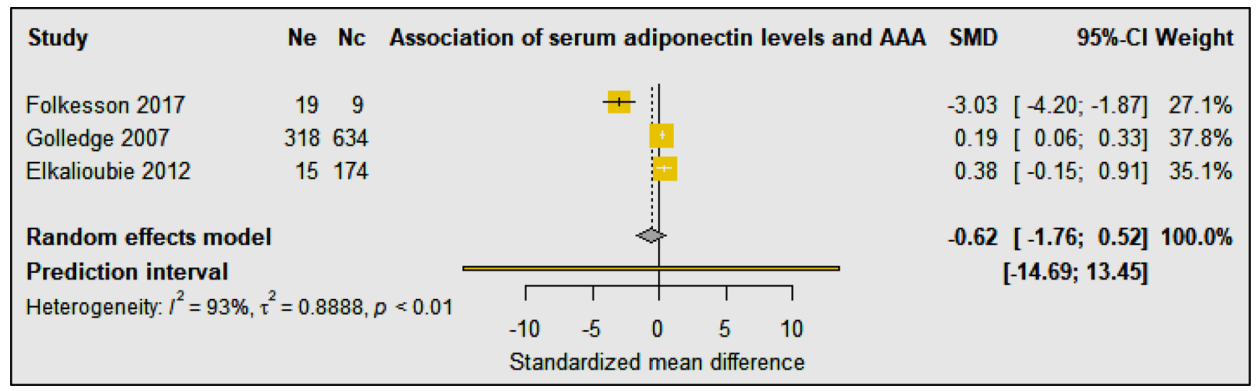

FIGURE 7 | Forest plot suggesting no association of circulating adiponectin concentrations with AAA diagnosis. Ne, Number of patients in experimental group; Nc, Number of patients in control group.

adjusting for confounders $(31,33,34)$. Three studies reported the methods used for aortic diameter imaging $(30,32,34)$. Two studies reported the methods used for aortic diameter assessment $(32,34)$. One study imaged both AAA cases and the comparator groups (34). One study reported the sample size estimates used (31).

\section{Studies Examining the Association of PVAT With AAA}

Three studies were identified investigating peri-aortic and visceral adipose tissue in 3,337 AAA cases compared with 382 control participants (37-39) (Table 4). The design of the studies included retrospective, prospective, and longitudinal cohort and case-control studies. All three studies matched the AAA cases and comparators for age and sex (37-39). In a multicenter retrospective case-control study, comparison of participants with asymptomatic AAA, aortoiliac occlusive disease (AIOD), and healthy controls suggested that AAA was associated with higher PVAT density and the visceral to subcutaneous adipose tissue (VAT/SAT) ratio was significantly greater in AAA patients (37). This association persisted after adjustment for cardiovascular risk factors and other diseases. Another casecontrol study compared AAA and intermittent claudication control subjects and also reported that the visceral to total abdominal adipose volume ratio was significantly greater in participants diagnosed with AAA, but the association was abolished after adjusted for age, heart disease, diabetes, smoking, sex, and hypertension (38). In a Framingham study including 3,001 individuals, the authors reported a significant association between peri-aortic fat deposition and larger aortic dimensions after adjusting for age, sex, cardiometabolic risk factors, and BMI, further supporting the notion that fat depots present around the aorta may contribute to its remodeling (39). Meta-analysis suggested that PVAT to abdominal adipose tissue ratio was significantly associated with AAA diagnosis (SMD: 0.56 [95\% CI 0.33, 0.79]) (Figure 8).

\section{Quality of Human Studies Investigating the Association of PVAT With AAA}

All studies reported the methods used to image the aorta, methods used for aortic diameter assessment and adjusted for potential confounders during analysis (Table 4) (37-39). Two studies reported the sample size estimate used $(37,38)$. Only one study blinded the observer during analysis of experimental data (37).

\section{Overall Quality Assessment of All Included Human Studies}

Overall, two $(37,38)$, two $(34,39)$, and four $(30-33)$ of the clinical research studies were considered to have low, moderate, and high risk of bias respectively (Table 5).

TABLE 4 | Examples of clinical studies investigating the association of perivascular adipose tissue with AAA.

\begin{tabular}{|c|c|c|c|c|c|c|c|}
\hline Reference & Study design & Groups & $\begin{array}{l}\text { Sample } \\
\text { size }\end{array}$ & Aortic diameter & PVAT to AAT ratio & $\begin{array}{c}p \\
\text { value }\end{array}$ & Confounders adjusted for analyses \\
\hline$(37)$ & $\begin{array}{l}\text { Retrospective } \\
\text { case control } \\
\text { study }\end{array}$ & $\begin{array}{l}\text { Control } \\
\text { AIOD } \\
\text { aAAA }\end{array}$ & $\begin{array}{c}97 \\
104 \\
140\end{array}$ & $\begin{array}{l}2.0 \pm 0.2^{\$}(\mathrm{~cm}) \\
2.1 \pm 0.4^{\$}(\mathrm{~cm}) \\
6.1 \pm 1.4^{\$}(\mathrm{~cm})\end{array}$ & $\begin{array}{l}1.2 \pm 0.7^{\star} \\
1.2 \pm 0.8^{\star} \\
1.5 \pm 0.7^{\star}\end{array}$ & 0.006 & $\begin{array}{l}\text { Gender, diabetes, hypertension, smoking, CHD, } \\
\text { PAD, BMl, anticoagulation, antiplatelet therapy, } \\
\text { vasodilator, diuretics, CCB, BB, statins }\end{array}$ \\
\hline$(38)$ & $\begin{array}{l}\text { Case control } \\
\text { study }\end{array}$ & $\begin{array}{l}\text { AAA } \\
\text { IC }\end{array}$ & $\begin{array}{l}196 \\
181\end{array}$ & $\begin{array}{l}50.0(42.0-57.0)^{\$}(\mathrm{~mm}) \\
21.0(20.0-24.0)^{\$}(\mathrm{~mm})\end{array}$ & $\begin{array}{l}0.5(0.4,0.6)^{\star \star} \\
0.4(0.3,0.5)^{\star \star}\end{array}$ & 0.007 & $\begin{array}{l}\text { Age, CHD, diabetes, ever smoked, sex and } \\
\text { hypertension }\end{array}$ \\
\hline$(39)^{\delta}$ & $\begin{array}{l}\text { Framingham } \\
\text { Heart - } \\
\text { Longitudinal } \\
\text { cohort study }\end{array}$ & $\begin{array}{l}\text { AAA in } \\
\text { women } \\
\text { AAA in } \\
\text { men }\end{array}$ & $\begin{array}{l}1,474 \\
1,527\end{array}$ & $\begin{array}{l}17 \pm 2^{\$}(\mathrm{~mm}) \\
19 \pm 2^{\$}(\mathrm{~mm})\end{array}$ & $\begin{array}{l}\text { HR }(95 \% \mathrm{Cl}): \\
\text { VAT }-0.3(0.2 \text { to } 0.4) \delta \\
\text { AAT }-0.3(0.2 \text { to } 0.3) \delta\end{array}$ & $<0.01$ & Age, sex, cardiometabolic risk factors, and BMI \\
\hline
\end{tabular}

AAA, Abdominal aortic aneurysm; aAAA, Asymptomatic AAA; AAT, Abdominal peri-aortic fat; AIOD, Aortoliac occlusive disease; BB, Beta blockers; BMI, Body mass index; CCB, Calcium channel blockers; $C H D$, Coronary heart disease; Cl, Confidence interval; cm, Centimeter; HR, Hazard ratio; IC, Intermittent claudication; mm, Millimeter; PAD, Peripheral artery disease; PVAT, Perivascular adipose tissue; VAT/SAT, Visceral adipose tissue/subcutaneous adipose tissue; *Data reported as mean \pm Standard deviation; ${ }^{* *}$ Data reported as median with $25^{\text {th }}$ and $75^{\text {th }}$ percentile range; $\delta$, Data reported as hazard ratio with 95\% confidence interval; ${ }^{\$}$ Aortic diameter measured using Computed tomography (CT). 


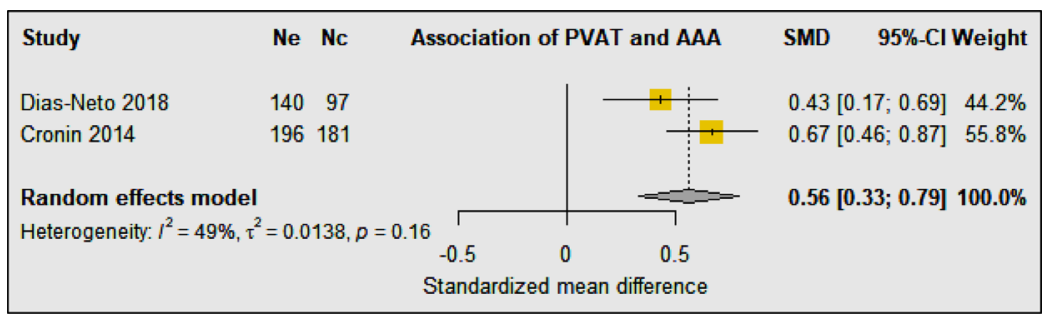

FIGURE 8 | Forest plot suggesting a significant association between greater perivascular adipose tissue to abdominal adipose tissue ratio and AAA diagnosis. Ne, Number of patients in experimental group; Nc, Number of patients in control group.

TABLE 5 | Quality assessment of clinical studies investigating the role of adipokines and PVAT in AAA.

\begin{tabular}{|c|c|c|c|c|c|c|c|c|c|c|}
\hline Reference & $\begin{array}{l}\text { Sample size } \\
\text { estimate } \\
\text { reported }\end{array}$ & $\begin{array}{c}\text { Age } \\
\text { matched } \\
\text { controls }\end{array}$ & $\begin{array}{c}\text { Sex } \\
\text { matched } \\
\text { controls }\end{array}$ & $\begin{array}{l}\text { Controls and } \\
\text { AAA cases } \\
\text { imaged }\end{array}$ & $\begin{array}{l}\text { Method of } \\
\text { aortic diameter } \\
\text { imaging }\end{array}$ & $\begin{array}{l}\text { Method of AAA } \\
\text { diameter } \\
\text { assessment }\end{array}$ & $\begin{array}{l}\text { Analysis by } \\
\text { blinded } \\
\text { observer }\end{array}$ & $\begin{array}{l}\text { Confounders } \\
\text { were adjusted for } \\
\text { analyses }\end{array}$ & Total & $\begin{array}{c}\text { Quality } \\
\text { score } \\
(\%)\end{array}$ \\
\hline (31) & 1 & 0 & 0 & 0 & 0 & 0 & 0 & 1 & 2 & 25 \\
\hline (33) & 0 & 1 & 0 & 0 & 0 & 0 & 0 & 1 & 2 & 25 \\
\hline (34) & 0 & 1 & 1 & 1 & 1 & 1 & 0 & 1 & 6 & 75 \\
\hline (30) & 0 & 0 & 0 & 0 & 1 & 0 & 0 & 0 & 1 & 12.5 \\
\hline (39) & 0 & 1 & 1 & 0 & 1 & 1 & 0 & 1 & 5 & 62.5 \\
\hline
\end{tabular}

AAA, Abdominal aortic aneurysm; PVAT, Perivascular adipose tissue; \%, Percentage; Yes $=1 ;$ No $=0$; Score of $8=100 \%$.

\section{CONCLUSIONS}

Overall this systematic review suggests inconsistent effects of adipokines and PVAT on AAA development within rodent AAA models. Circulating levels of leptin and resistin and PVAT to abdominal adipose tissue ratio, but not adiponectin, were found to be significantly greater in people diagnosed with AAA compared to controls. Given the limited number of eligible animal and human studies, more evidence is needed before any robust conclusions about the role of adipokines and PVAT in AAA pathogenesis can be made. The quality assessments suggested that this past research had a moderate risk of bias.

\section{DATA AVAILABILITY STATEMENT}

The original contributions presented in the study are included in the article/supplementary material. Further inquiries can be directed to the corresponding author.

\section{REFERENCES}

1. Svensjö S, Björck M, Wanhainen A. Update on Screening for Abdominal Aortic Aneurysm: A Topical Review. Eur J Vasc Endovasc Surg (2014) 48 (6):659-67. doi: 10.1016/j.ejvs.2014.08.029

2. Liu B, Granville DJ, Golledge J, Kassiri Z. Pathogenic mechanisms and the potential of drug therapies for aortic aneurysm. Am J Physiol Heart Circulatory Physiol (2020) 318(3):H652-h70. doi: 10.1152/ajpheart.00621.2019

3. Golledge J. Abdominal aortic aneurysm: update on pathogenesis and medical treatments. Nat Rev Cardiol (2019) 16(4):225-42. doi: 10.1038/s41569-018-0114-9

\section{AUTHOR CONTRIBUTIONS}

JG conceptualized the study. ST performed the screening of studies, data extraction, and analysis. Both ST and JG contributed to preparation of the manuscript. JG critically assessed the manuscript. All authors contributed to the article and approved the submitted version.

\section{FUNDING}

Funding from the National Health and Medical Research Council (1063476 and 1022752), James Cook University, The Townsville Hospital and Health Services Study, Education and Research Trust Fund, and Queensland Government supported this work. Jonathan Golledge holds a Practitioner Fellowship from the National Health and Medical Research Council (1117061) and a Senior Clinical Research Fellowship from the Queensland Government, Australia.

4. Filardo G, Powell JT, Martinez MA-M, Ballard DJ. Surgery for small asymptomatic abdominal aortic aneurysms. Cochrane Database Syst Rev (2012) 3(3):CD001835. doi: 10.1002/14651858.CD001835.pub3

5. United Kingdom Small Aneurysm Trial P, Powell JT, Brady AR, Brown LC, Fowkes FG, Greenhalgh RM, et al. Long-term outcomes of immediate repair compared with surveillance of small abdominal aortic aneurysms. N Engl J Med (2002) 346(19):1445-52. doi: 10.1056/NEJMoa 013527

6. The UK Small Aneurysm Trial Participants. Mortality results for randomised controlled trial of early elective surgery or ultrasonographic surveillance for 
small abdominal aortic aneurysms. Lancet (London, England). (1998) 352:1649-55. doi: 10.1016/S0140-6736(98)10137-X

7. Golledge J, Moxon JV, Singh TP, Bown MJ, Mani K, Wanhainen A. Lack of an effective drug therapy for abdominal aortic aneurysm. J Internal Med (2020) 288(1):6-22. doi: 10.1111/joim.12958

8. Hajer GR, van Haeften TW, Visseren FL. Adipose tissue dysfunction in obesity, diabetes, and vascular diseases. Eur Heart J (2008) 29(24):2959-71. doi: 10.1093/eurheartj/ehn387

9. Blüher M. Importance of adipokines in glucose homeostasis. Diabetes Manage (2013) 3(5):389. doi: 10.2217/dmt.13.35

10. Farooqi IS, Jebb SA, Langmack G, Lawrence E, Cheetham CH, Prentice AM, et al. Effects of recombinant leptin therapy in a child with congenital leptin deficiency. New Engl J Med (1999) 341(12):879-84. doi: 10.1056/NEJM199909163411204

11. Fasshauer M, Blüher M. Adipokines in health and disease. Trends Pharmacol Sci (2015) 36(7):461-70. doi: 10.1016/j.tips.2015.04.014

12. Ye R, Scherer PE. Adiponectin, driver or passenger on the road to insulin sensitivity? Mol Metab (2013) 2(3):133-41. doi: 10.1016/j.molmet.2013.04.001

13. Lamers D, Famulla S, Wronkowitz N, Hartwig S, Lehr S, Ouwens DM, et al. Dipeptidyl peptidase 4 is a novel adipokine potentially linking obesity to the metabolic syndrome. Diabetes (2011) 60(7):1917-25. doi: 10.2337/db10-1707

14. Jamaluddin MS, Weakley SM, Yao Q, Chen C. Resistin: functional roles and therapeutic considerations for cardiovascular disease. Br J Pharmacol (2012) 165(3):622-32. doi: 10.1111/j.1476-5381.2011.01369.x

15. Steppan CM, Bailey ST, Bhat S, Brown EJ, Banerjee RR, Wright CM, et al. The hormone resistin links obesity to diabetes. Nature (2001) 409(6818):307-12. doi: $10.1038 / 35053000$

16. Yang RZ, Huang Q, Xu A, McLenithan JC, Eisen JA, Shuldiner AR, et al. Comparative studies of resistin expression and phylogenomics in human and mouse. Biochem Biophys Res Commun (2003) 310(3):927-35. doi: 10.1016/ j.bbrc.2003.11.001

17. Kim HW, Chantemèle E, Weintraub NL. Perivascular Adipocytes in Vascular Disease. Arteriosclerosis Thrombosis Vasc Biol (2019) 39(11):2220-7. doi: 10.1161/ATVBAHA.119.312304

18. Piacentini L, Chiesa M, Colombo GI. Gene Regulatory Network Analysis of Perivascular Adipose Tissue of Abdominal Aortic Aneurysm Identifies Master Regulators of Key Pathogenetic Pathways. Biomedicines (2020) 8(8):288. doi: 10.3390/biomedicines8080288

19. Tao M, Yu P, Nguyen BT, Mizrahi B, Savion N, Kolodgie FD, et al. Locally applied leptin induces regional aortic wall degeneration preceding aneurysm formation in apolipoprotein E-deficient mice. Arteriosclerosis Thrombosis Vasc Biol (2013) 33(2):311-20. doi: 10.1161/ATVBAHA.112.300543

20. Liu CL, Ren J, Wang Y, Zhang X, Sukhova GK, Liao M, et al. Adipocytes promote interleukin-18 binding to its receptors during abdominal aortic aneurysm formation in mice. Eur Heart $J$ (2020) 41(26):2456-68. doi: 10.1093/eurheartj/ehz856

21. Police SB, Thatcher SE, Charnigo R, Daugherty A, Cassis LA. Obesity promotes inflammation in periaortic adipose tissue and angiotensin IIinduced abdominal aortic aneurysm formation. Arteriosclerosis Thrombosis Vasc Biol (2009) 29(10):1458-64. doi: 10.1161/ATVBAHA.109.192658

22. Zhang Y, Yuan H, Bu P, Shen YH, Liu T, Song S, et al. Recombinant leptin attenuates abdominal aortic aneurysm formation in angiotensin II-infused apolipoprotein E-deficient mice. Biochem Biophys Res Commun (2018) 503 (3):1450-6. doi: 10.1016/j.bbrc.2018.07.062

23. Wågsäter D, Vorkapic E, van Stijn CM, Kim J, Lusis AJ, Eriksson P, et al. Elevated Adiponectin Levels Suppress Perivascular and Aortic Inflammation and Prevent AngII-induced Advanced Abdominal Aortic Aneurysms. Sci Rep (2016) 6:31414. doi: 10.1038/srep31414

24. Yoshida S, Fuster JJ, Walsh K. Adiponectin attenuates abdominal aortic aneurysm formation in hyperlipidemic mice. Atherosclerosis (2014) 235 (2):339-46. doi: 10.1016/j.atherosclerosis.2014.05.923

25. Meng X, Zhang K, Kong J, Xu L, An G, Qin W, et al. Deletion of resistin-like molecule-beta attenuates angiotensin II-induced abdominal aortic aneurysm. Oncotarget (2017) 8(61):104171-81. doi: 10.18632/oncotarget.22042
26. Meng X, Kong J, Zhang K, Yang J, Li J, Zhang Y. Association of resistin-like molecule $\beta$ levels and abdominal aortic aneurysm. Int J Cardiol (2015) 181:810. doi: $10.1016 /$ j.ijcard.2014.11.197

27. Sakaue T, Suzuki J, Hamaguchi M, Suehiro C, Tanino A, Nagao T, et al. Perivascular Adipose Tissue Angiotensin II Type 1 Receptor Promotes Vascular Inflammation and Aneurysm Formation. Hypertens (Dallas Tex 1979) (2017) 70(4):780-9. doi: 10.1161/HYPERTENSIONAHA.117.09512

28. Kugo H, Moriyama T, Zaima N. The role of perivascular adipose tissue in the appearance of ectopic adipocytes in the abdominal aortic aneurysmal wall. Adipocyte (2019) 8(1):229-39. doi: 10.1080/21623945.2019.1636625

29. Schroeter MR, Eschholz N, Herzberg S, Jerchel I, Leifheit-Nestler M, Czepluch FS, et al. Leptin-Dependent and Leptin-Independent Paracrine Effects of Perivascular Adipose Tissue on Neointima Formation. Arteriosclerosis Thrombosis Vasc Biol (2013) 33(5):980-7. doi: 10.1161/ATVBAHA.113.301393

30. Folkesson M, Vorkapic E, Gulbins E, Japtok L, Kleuser B, Welander M, et al. Inflammatory cells, ceramides, and expression of proteases in perivascular adipose tissue adjacent to human abdominal aortic aneurysms. J Vasc Surg (2017) 65(4):1171-9.e1. doi: 10.1016/j.jvs.2015.12.056

31. Kubota Y, Folsom AR, Pankow JS, Wagenknecht LE, Tang W. Diabetesrelated factors and abdominal aortic aneurysm events: the Atherosclerotic Risk in Communities Study. Ann Epidemiol (2018) 28(2):102-6.e1. doi: 10.1016/j.annepidem.2017.12.003

32. Lu HY, Huang CY, Shih CM, Lin YW, Tsai CS, Lin FY, et al. A potential contribution of dipeptidyl peptidase- 4 by the mediation of monocyte differentiation in the development and progression of abdominal aortic aneurysms. J Vasc Surg (2017) 66(4):1217-26.e1. doi: 10.1016/ j.jvs.2016.05.093

33. Elkalioubie A, Juthier F, Gautier C, Vincentelli A, Pinet F, Jude B, et al. Resistin is independently associated with abdominal aortic aneurysm in severe coronary artery disease patients. Cardiovasc Pathol Off J Soc Cardiovasc Pathol (2012) 21(2):e27-9. doi: 10.1016/j.carpath.2011.06.001

34. Golledge J, Clancy P, Jamrozik K, Norman PE. Obesity, adipokines, and abdominal aortic aneurysm: Health in Men study. Circulation (2007) 116 (20):2275-9. doi: 10.1161/CIRCULATIONAHA.107.717926

35. Horimatsu T, Kim HW, Weintraub NL. The Role of Perivascular Adipose Tissue in Non-atherosclerotic Vascular Disease. Front Physiol (2017) 8:969. doi: $10.3389 /$ fphys.2017.00969

36. Li MW, Mian MO, Barhoumi T, Rehman A, Mann K, Paradis P, et al. Endothelin-1 overexpression exacerbates atherosclerosis and induces aortic aneurysms in apolipoprotein E knockout mice. Arteriosclerosis Thrombosis Vasc Biol (2013) 33(10):2306-15. doi: 10.1161/ATVBAHA.113.302028

37. Dias-Neto M, Meekel JP, van Schaik TG, Hoozemans J, Sousa-Nunes F, Henriques-Coelho T, et al. High Density of Periaortic Adipose Tissue in Abdominal Aortic Aneurysm. Eur J Vasc Endovasc Surg Off J Eur Soc Vasc Surg (2018) 56(5):663-71. doi: 10.1016/j.ejvs.2018.07.008

38. Cronin O, Liu D, Bradshaw B, Iyer V, Buttner P, Cunningham M, et al. Visceral adiposity is not associated with abdominal aortic aneurysm presence and growth. Vasc Med (London England) (2014) 19(4):272-80. doi: 10.1177/ $1358863 X 14537883$

39. Thanassoulis G, Massaro JM, Corsini E, Rogers I, Schlett CL, Meigs JB, et al. Periaortic adipose tissue and aortic dimensions in the Framingham Heart Study. J Am Heart Assoc (2012) 1(6):e000885. doi: 10.1161/JAHA.112.000885

Conflict of Interest: The authors declare that the research was conducted in the absence of any commercial or financial relationships that could be construed as a potential conflict of interest.

Copyright (C) 2021 Thanigaimani and Golledge. This is an open-access article distributed under the terms of the Creative Commons Attribution License (CC BY). The use, distribution or reproduction in other forums is permitted, provided the original author(s) and the copyright owner(s) are credited and that the original publication in this journal is cited, in accordance with accepted academic practice. No use, distribution or reproduction is permitted which does not comply with these terms. 